\title{
INFLUENCE OF THE HYDROFOIL INCLINATION ANGLE AT FREE SURFACE FLOW AROUND JUNCTIONS
}

\author{
Costel Ungureanu \\ ${ }^{1}$ University "Dunarea de Jos" of Galati, \\ Faculty of Naval Architecture, Galati, Domneasca \\ Street, No. 47, 800008, Romania, \\ E-mail:costel.ungureanu@ugal.ro \\ ${ }^{2}$ Bureau Veritas Romania \\ 165 Splaiul Unirii Street, TN Offices, 3rd Building, \\ B Wing, 5th Floor, 3rd District, 030133, \\ Bucharest, Romania, \\ E-mail:costel.ungureanu@bureauveritas.com
}

\author{
Costel Iulian Mocanu \\ University "Dunarea de Jos" of Galati, \\ Faculty of Naval Architecture, Galati, Domneasca \\ Street, No. 47, 800008, Romania, \\ E-mail:costel.mocanu@ugal.ro
}

\begin{abstract}
Free surface flow is a hydrodynamic problem with a seemingly simple geometric configuration but with a flow topology complicated by the pressure gradient due to the presence of the obstacle, the interaction between the boundary layer and the free surface, turbulence, breaking waves, surface tension effects between water and air. As the ship appendages become more and more used and larger in size, the general understanding of the flow field around the appendages and the junction between them and the hull is a topical issue for naval hydrodynamics. When flowing with a boundary layer, when the streamlines meet a bluff body mounted on a solid flat or curved surface, detachments appear in front of it due to the blocking effect. As a result, vortex structures develop in the fluid, also called horseshoe vortices, the current being one with a completely three-dimensional character, complicated by the interactions between the boundary layer and the vortex structures thus generated. Despite the importance of the topic, the literature records the lack of coherent methods for investigating free surface flow around junctions, the lack of consistent studies on the influence of the inclination of the profile mounted on the body. As a result, this paper aims to systematically study the influence of profile inclination in respect to the support plate.
\end{abstract}

Keywords: free surface flow, junction flow, RANS, VOF, open channel flow

\section{INTRODUCTION}

Free surface flow around a hydrodynamic profile mounted on a plate near the free surface is a complicated one that combines nonlinear, three-dimensional and turbulent phenomena such as the boundary layer on solid surfaces, the horseshoe vortex system developed around junctions and the effects of the free surface, as if turbulent, breaking the wave. As a result, this paper presents the results of the numerical study on free surface flow around junctions, more precisely the study of the influence of the angle between profile and plate by inclining the profile in three directions: lateral, upstream and downstream, with three steps, 15,30 and 45 degrees, starting from the upright position. Due to the complexity of the discretization grid generated on a computational domain with constraints imposed by numerical resolution of the free surface in the Ansys Fluent program, numerical simulations were performed 
only for the junction between the hydrodynamic profile and the flat plate, following in perspective with the development of open channel flow from.

\section{NUMERICAL MODEL}

\subsection{Boundary Conditions}

When flowing with a free surface, the surface piercing hydrofoil generates a divergent waves system propagating at an angle of approximately $20^{\circ}$. In order to avoid the radiation of the waves upstream, the entrance boundary is positioned at 1.5 chord lengths upstream of the profile. In order to avoid the reflection of the wave system from the lateral boundaries, producing numerical instabilities, the width of the calculation domain as well as the length of the downstream area are established so that the wave system leaves the integral domain through the downstream boundary. As a result, the downstream boundary is arranged at five chord lengths from the profile trailing edge, and the side boundaries at two 2.5 chord lengths from the profile plane of symmetry. The position of the upper boundary is chosen so as to allow the natural elevation of the free surface (figure 1). Initial tests established that 0.5 of the profile chord length is sufficient.

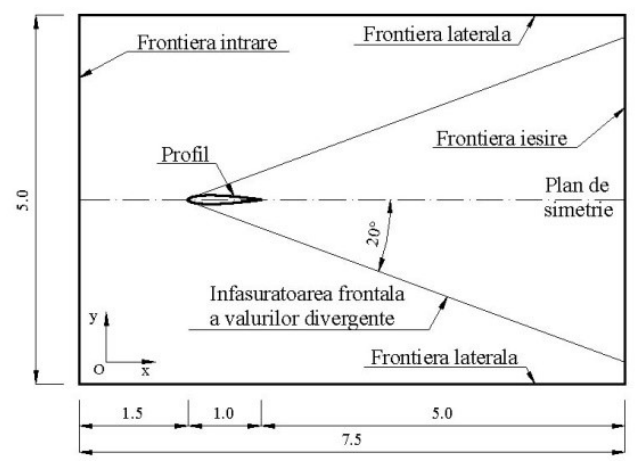

Fig.1. Calculation model dimensions

Free surface flow is governed by gravitational force and inertial forces, represented by the dimensionless parameter Froude. As a result, the boundary conditions imposed must take into account the effects of gravity. In this sense, the calculation domain is modeled for open channel flow. Figure 2 shows the computational domain together with the boundary conditions imposed. The inlet and outlet boundaries of the fluid in the field are defined as pressure inlet and outlet boundaries. On these, the level of the undisturbed free surface is declared, as well as the elevation of the bottom for the solver to calculate the hydrostatic pressure. Also, the fluid velocity at infinite upstream and the initial turbulent sizes are declared.

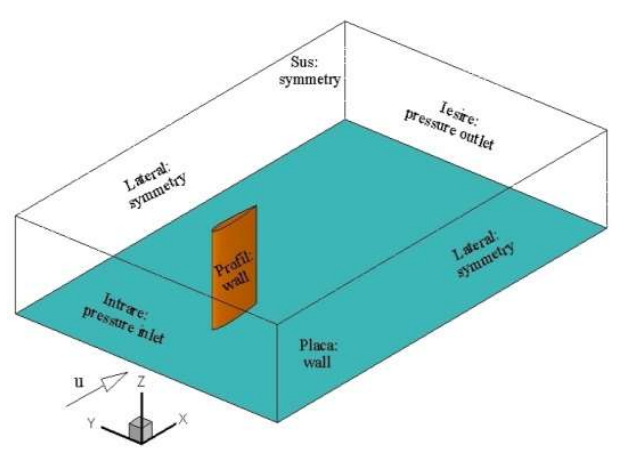

Fig.2. Boundary conditions

In the case of the $k-\omega \mathrm{SST}$ model, the turbulence intensity, calculated on the basis of the hydraulic diameter, and the ratio between the turbulence and the molecular viscosity shall be declared. In the problems with the free surface, the calculation range is defined from a single volume, following that after the initialization of the simulation, the areas for the two fluids water and air will be declared. On the profile and on the plate, the wall-type no-slip condition is required. The symmetry condition is declared on the side boundaries as well as on the upper boundary of the calculation range. In the present study, the explicit, quasi-stationary calculation with advancement in time, proved to be a much more robust and stable alternative than the stationary calculation. Fluent's calculation algorithm is quite robust and allows advancement over time without distorting the results. In order not to introduce errors in the calculation, when integrating the flows on the cells, the time step necessary to integrate 
the flow equations is calculated based on the volume of the smallest cell in the discretization network.

\subsection{Numerical schemes}

The system of RANS equations together with the equations of the $k-$ SST turbulence model are solved in a non-stationary regime, in a quasi-explicit manner, using the finite volume method from Ansys Fluent. The gradients of the solution are obtained by applying the cell-based Green-Gauss theorem. Solving problems with the free surface in the Fluent program is done by the VOF method and is solved using the geometric reconstruction scheme. The coupling of speed and pressure is solved by the PISO algorithm, imposed by the unsteady calculation. The pressure is described by the PRESTO scheme, and the convective and diffusive terms are solved by the QUICK scheme.

\section{3. . Grid Generation}

The boundary conditions implemented in Fluent for open channel flow impose vertical boundaries for both inlet and outlet. Figure 3 shows the calculation range for the case of the profile inclined by 30 degrees in the downward symmetry plane where the delimitation lines of the component grids can be observed.

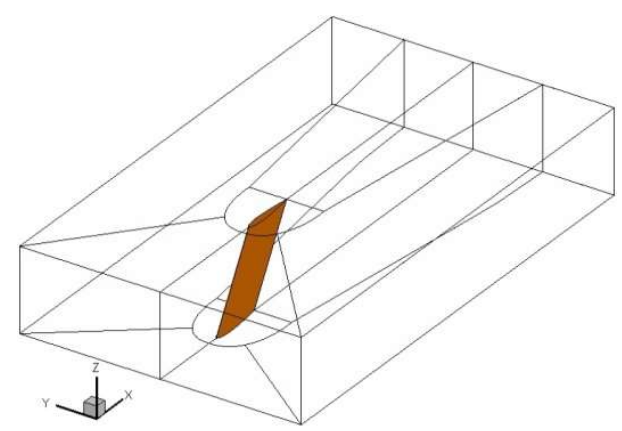

Fig.3. Multiblock calculation model

The flow around the junction is described by phenomena such as the interaction

(C) Galati University Press, 2020 of two boundary layers, one on the plate and the other on the profile. At the same time the grid must meet quality conditions such as spacing and orthogonality on solid boundaries. In addition, if the free surface is added, the discretization for solving by the VOF method must describe a band of condensed nodes in the area where the formation of the wave system is expected. Taking into account all the conditions imposed for solving the imposed physical problems, a structured multiblock grid is generated that perfectly overlaps the rectangular computing range, ensuring orthogonal and condensed grid lines on the base plate and on the hydrodynamic profile but which at the same time ensures the minimum space necessary to capture the elevation of the free surface. This results in a discretization grid with approximately two million hexahedral cells. Figure 4 shows the discretized computing domain in structured multiblock style of type C-H.

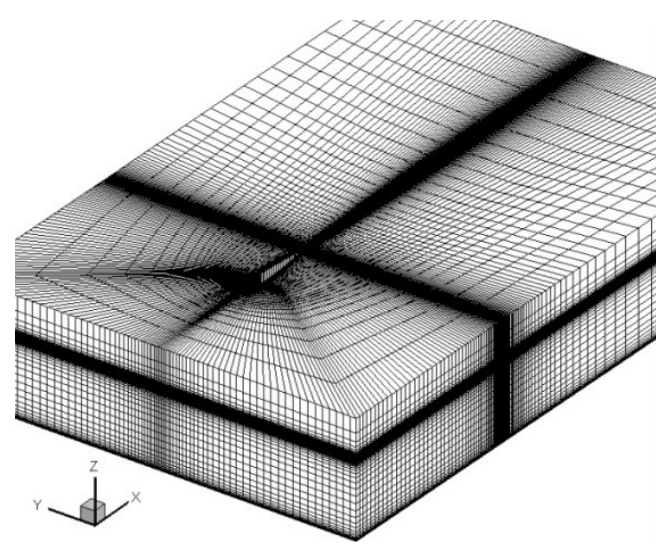

Fig.4. C-H tridimensional grid

\section{RESULTS AND DISCUSSIONS}

The influence of the angle between the profile and the plate is studied by inclining the hydrofoil in three directions: lateral, upstream and downstream, with three steps, 15, 30 and 45 degrees, starting from the position of the upright vertical profile, constantly keeping the profile chord parallel to the direction flow. Together with the case of the right vertical profile it forms the set of geo- 
metric hypostases of the profile in relation to the base plate.

The following are the vortex structures that develop at the meeting of streamlines with the leading edge of the hydrofoil due to the blocking phenomenon. Figure 5 shows the system of two counter-rotating vortices, one main of higher intensity and one secondary of smaller intensity located in the vicinity of the leading edge of the profile as well as the evolution in relation to the inclination of the profile in the normal direction to the vertical plane of symmetry. The influence of the base plate is observed as the profile tilts, causing the main vortex to move towards the hydrodynamic profile.

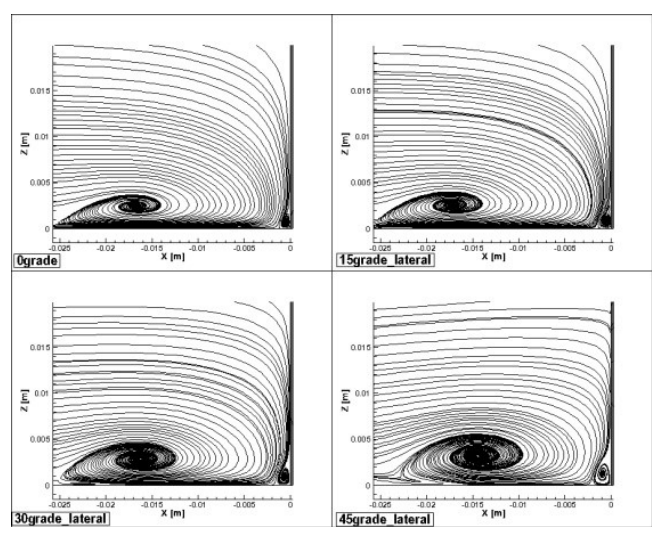

Fig.5. Streamlines -comparison between vertical and laterally inclined hydrofoil

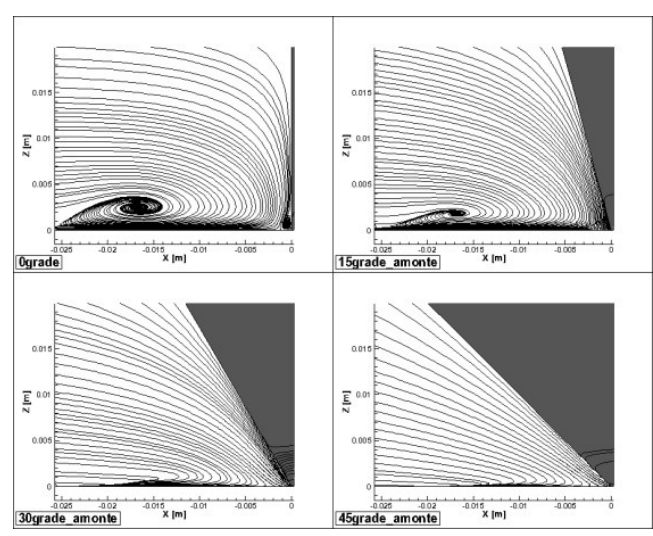

Fig. 6. Streamlines -comparison between vertical and upstream inclined hydrofoil
In the upstream inclined profile (figure 6) it is observed that the main vortex moves towards the base plate and due to the high pressure gradients generated by the presence of the two solid surfaces, at 30 and 45 degrees the vortex structures are no longer formed.

When the profile is inclined downstream, the current lines approach the leading edge of the profile leading to a ring of the position of the main vortex with the secondary one, together with the increase in intensity and diameter of the core of the main vortex. If at the right vertical profile, the secondary vortex is in the vicinity of the leading edge, at 30 and 45 degrees it moves away from the leading profile.

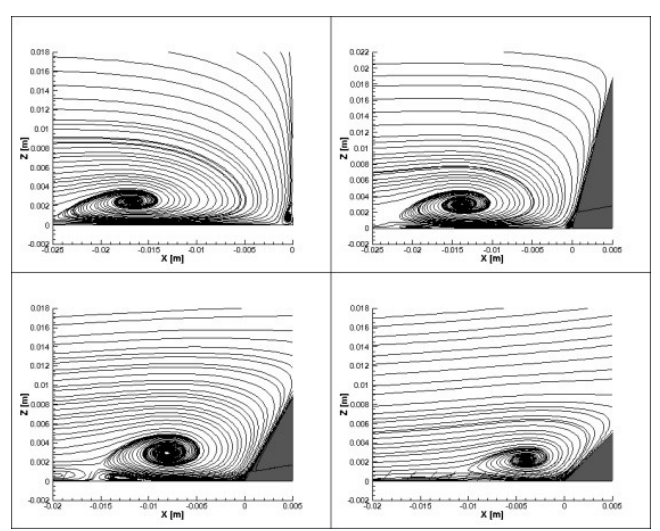

Fig. 7. Streamlines -comparison between vertical and downstream inclined hydrofoil

It can also be seen that if at the vertical profile the presence of the profile leads the current lines in the downstream direction, the large angle of inclination allows the streamlines to slide in the horizontal and upstream direction. This allows a reduction of the pressure gradient and thus facilitates the translation of the main vortex to the hydrodynamic profile.

Figures 8 and 9 show the evolution of the free surface in relation to the angle of inclination of the profile to the side. It is observed that the geometric asymmetry induces differences between the two edges of the profile and of the generated wave system, 
asymmetry in the flow field, but also a phase shift of the crest / trough alternation

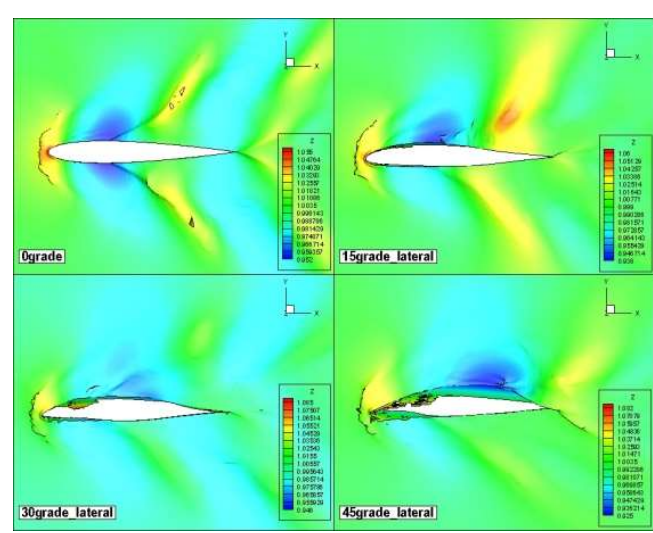

Fig. 8. Free surface -comparison between vertical and laterally inclined hydrofoil, top view

The yellow and red colors show the wave crests and the blue show the wave through. There is also an increase in the size of the wave crest as well as a deepening of the wave trough as the angle of inclination increases. The numerical free surface was generated as iso-surface of the water-air volume fraction equal to 0.5 .

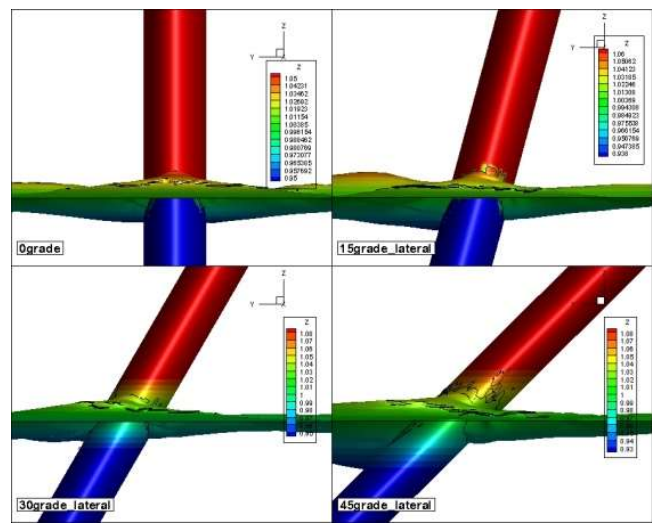

Fig. 9. Free surface -comparison between vertical and laterally inclined hydrofoil, front view

Figure 10 shows the evolution of the free surface in relation to the angle of inclination of the downstream profile. It is observed that as the angle of inclination increases, the am- plitude of the first wave generated by the profile increases.

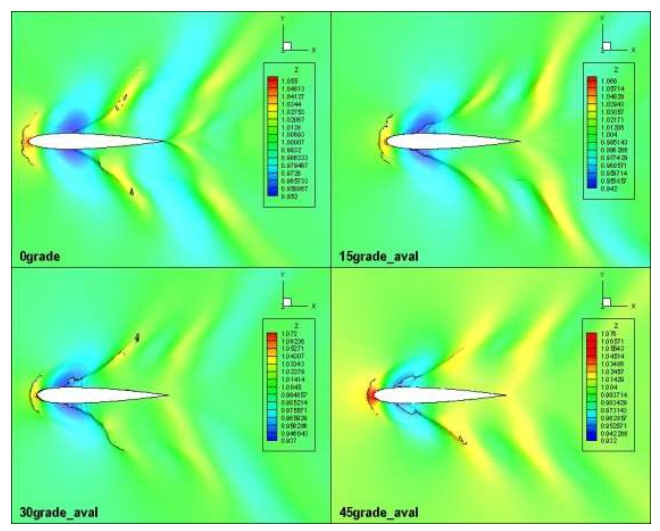

Fig. 10. Free surface -comparison between vertical and downstream inclined hydrofoil, top view

Figure 11 shows the evolution of the free surface in relation to the strut inclination angle toward upstream. It is observed that the amplitude of the first wave generated by the profile decreases with increasing angle of inclination, because the streamlines are driven downwards, similar to the junction at the base of the profile. If in the case of the upright profile the second wave crest is prominent, it decreases in amplitude as the angle between the plate and the profile increases.

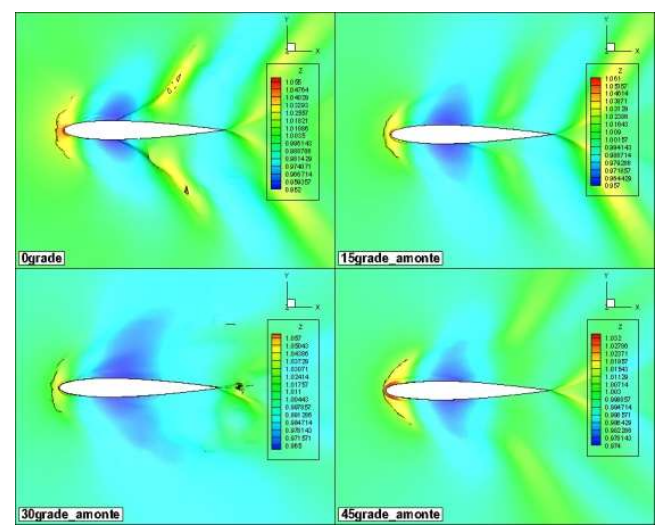

Fig. 11. Free surface -comparison between vertical and upstream inclined hydrofoil, top view 
The drag coefficients were calculated and presented in Figure 12, for the Fn $=0.32$ case.. There is a decrease of up to $10 \%$ of the total coefficient in the case of lateral inclination and a decrease of up to $55 \%$ in the case of inclination in the upstream and downstream diametrical plane.

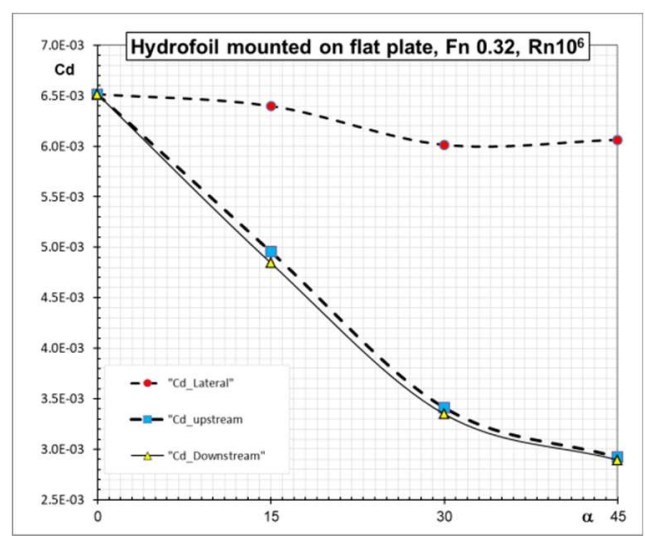

Fig. 12. Drag coefficients in respect to inclination angle

\section{CONCLUDING REMARKS}

In this chapter, a systematic numerical study of the flow around the junctions was presented and the influence of factors such as profile inclination, current speed and the influence of depth on the flow around the junction between a hydrodynamic profile and a flat plate was studied. The results were presented for inclination of the profile in 3 directions with three angle steps, 15,30 and 45 degrees in relation to the upright profile, mounted on a flat base plate.

Following the analysis of the numerical results of this systematic study, it is recom- mended that from a hydrodynamic point of view the mounting of the profile on the hull shall be made with an inclination upstream/downstream with maximum 15 degrees laterally inclination angle for the horshoe vortices to be diminished in intensity and the the profile drag values to be minimum in the global economy of the ship.

\section{Acknowledgements}

This research study was performed in the frame of Multidisciplinary Research Platform ReForm Dunarea de Jos University of Galati - The Naval Architecture Research Center.

\section{REFERENCES}

[1]. Fleming J., Simpson R.L., Devenport W.J. "An experimental study of a turbulent wingbody junction flow”, Experiments Fluids, 14:366-78, 1993.

[2]. Ungureanu, C., Lungu, A., „Numerical Studies on Free Surface Flow around a Hydrofoil Mounted on a Plate", Numerical Analysis and Applied Mathematics, AIP Proceedings, Melville New York, Vol. 1281, pp. 115-118, 2010.

[3]. Metcalf, B., et al., "Unsteady free surface wave-induced boundary-layer separation for a surface-piercing NACA 0024 foil: towing tank experiments", Journal of Fluids and Structures, 22, 77-98, 2006.

[4]. Ungureanu, C., ,, Towing Tank Experiments for a Surface Piercing NACA 0012 Hydrofoil", Annals of "Dunarea de Jos" University Galati. Fascicle XI, Shipbuilding, pp. 5-10, 2011;

Paper received on 15 of August, 2020 